\title{
Trigger techniques for B-Physics at CMS
}

\author{
Alessio Boletti*, on behalf of the CMS Collaboration \\ Universita' degli studi di Padova \& INFN \\ E-mail: alessio.boletti@cern.ch
}

The large instantaneous luminosity delivered by LHC to the CMS experiment in Run 2 is an important opportunity for the study of rare $\mathrm{B}$-hadron decays, like $\mathrm{B}_{(s)}^{0} \rightarrow \mu \mu$ and $\mathrm{B}^{0} \rightarrow \mathrm{K}^{* 0} \mu \mu$. The main drawback of this luminosity increase is that the trigger selections need to be tighter, in order to fit the rate of recorded events in the limits imposed by the available computer resources. This constraint imposes a challenge in the development of new trigger tools and algorithms, optimized to keep high acceptance on signal events while reducing the rate of collected background events, both at Level-1 Trigger (L1T) and at High-Level Trigger (HLT).

The International Conference on B-Physics at Frontier Machines - BEAUTY2018

6-11 May, 2018

La Biodola, Elba Island, Italy

* Speaker. 


\section{The CMS trigger system}

Events of interest are selected using a two-tiered trigger system [1]. The first level (L1T), composed of custom hardware processors, uses information from the calorimeters and muon detectors to select events at a rate of around $100 \mathrm{kHz}$ within a time interval of less than $4 \mu \mathrm{s}$. The second level, known as the high-level trigger (HLT), consists of a farm of processors running a version of the full event reconstruction software optimised for fast processing, and reduces the event rate to around $1 \mathrm{kHz}$ before data storage.

\section{Level-1 Trigger}

Triggering B-Physics events at L1T presents many challenges, due to the absence of information from the silicon detector:

- no dimuon vertexing prevents the rejection of events coming from different $p p$ collisions (pileup);

- no rejection of muons produced in a pion or kaon decay inside the tracker volume.

In the past years, many upgrades have been made in the L1T muon reconstruction criteria, in order to reduce the contamination from spurious candidates, generated by fake detector signals, punchthrough muons, or sail-through hadrons, and to improve the resolution of the kinematical variables assigned to the muon candidate [3].

Two important improvements, introduced before the 2017 data taking, are the possibility to use the muon $\eta$ and $\phi$ coordinates extrapolated to the interaction point, taking into account the curved trajectory due to the magnetic field, and the possibility to perform selections involving variables related to muon pairs, instead of single-muon quantities only. They allowed to add new selection criteria to the B-Physics triggers:

- A selection on $\Delta R_{\mu \mu}$, used for triggers selecting events with dimuon masses lower than 6 $\mathrm{GeV}$, for which the opening angle of the muons is limited. The upper threshold of this variable has been set to 1.2 and 1.4 for the two triggers used for these channels, accepting muons with $|\eta|<2.4$ and $|\eta|<1.5$, respectively. These threshold values are tuned in order to assure a complete acceptance on signal events, and not to alter the shape of the distributions of signal and sideband events. The efficiency of the selection $\Delta R_{\mu \mu}<1.2$ measured on a sample of $J / \psi$ events is shown in Figure 1.

- A selection on the dimuon invariant mass, used for triggers selecting events in the $\Upsilon$ mass range. The mass window set for the $\mathrm{L} 1$ triggers ranges from $8 \mathrm{GeV}$ to $17 \mathrm{GeV}$, to guarantee a very large acceptance over the whole mass window selected at HLT. The distribution of the dimuon mass variable as computed at L1T and compared with the one computed offline, for a sample of $\Upsilon$ candidates and mass-sideband events, is shown in Figure 2. 


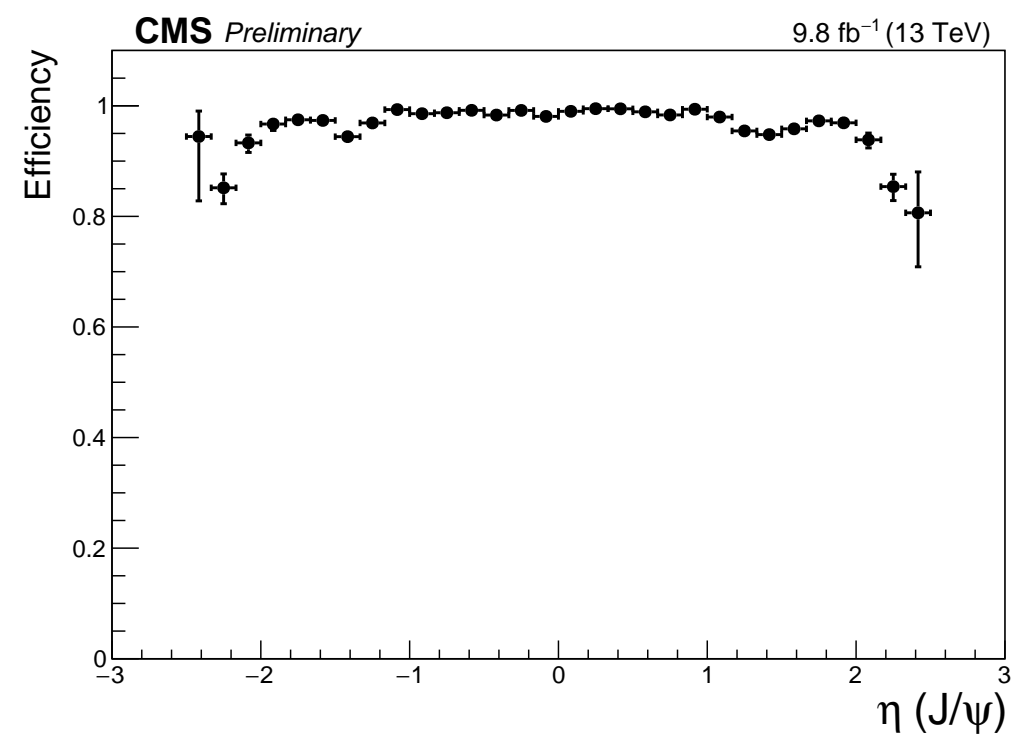

Figure 1: The efficiency of the Level-1 Trigger requirement $\Delta R_{\mu \mu}<1.2$ is shown against the pseudorapidity of the $J / \psi$ candidate. Figure from [5].

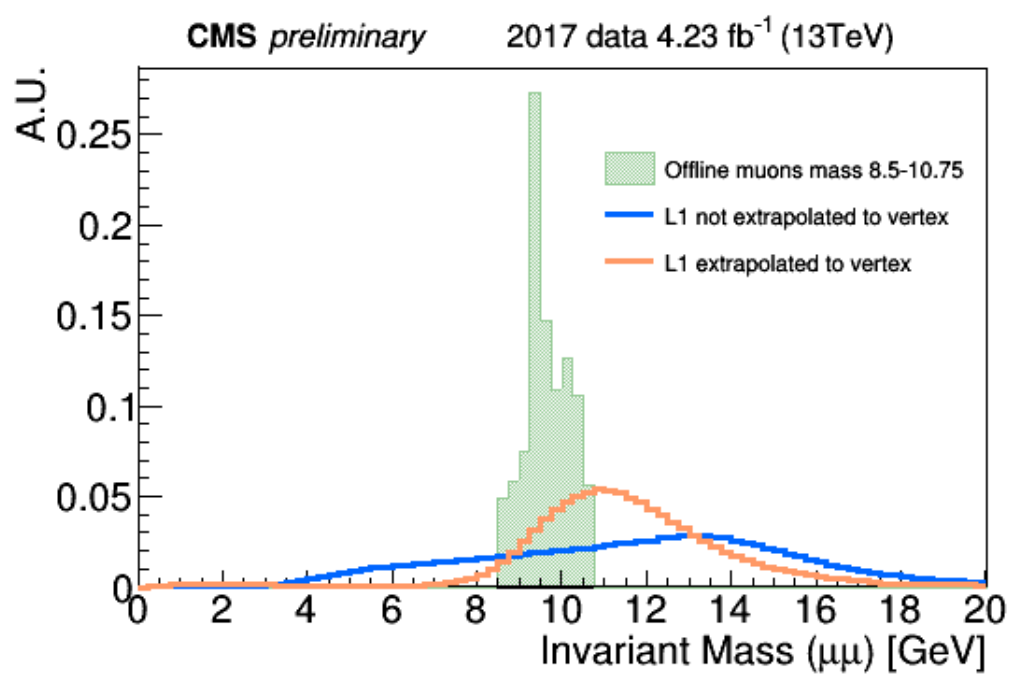

Figure 2: The $\mathrm{M}^{\text {offl }}$ spectrum with a cut at $8.5<\mathrm{M}^{\text {offl }}<10.75 \mathrm{GeV}$ and the $\mathrm{M}^{\mathrm{L} 1}$ spectrum of the L1 muons that match the offline muons in this interval. The L1 track extrapolation to the vertex improves the L1 dimuon resolution. Figure from [4]. 


\section{High-Level Trigger}

At HLT, the reconstruction of muon candidates is performed using both the information from muon chambers and silicon detector. This leads to a better rejection of fake muons and muons from pion and kaon decays. In addition, performing the vertexing between the muons allows to select candidates originating from the same interaction point, or from the same decay point.

The triggers used for B-Physics decay channels can be divided in two categories:

inclusive triggers, requiring a pair of muons (and, for some triggers, one or two additional muons) with the invariant mass compatible with one resonant state $\left(\phi, \psi, B_{(s)}^{0}\right.$, or $\left.\Upsilon\right)$ and with a vertexing requirement, without applying criteria on the vertex position with respect to the interaction point. The efficiency of the vertexing requirement, computed on a sample of $J / \psi$ candidates, is shown in Figure 3.

displaced triggers, requiring a pair of muons and an additional object (a track to select B hadron decays, or a muon to select candidates for $\tau \rightarrow 3 \mu$ searches). The invariant mass of the muon pair is required to be compatible with $J / \psi$ or $\psi(2 S)$ states, or in a non-resonant window in the range from $0.2 \mathrm{GeV}$ to $4.8 \mathrm{GeV}$. A vertexing requirement is applied, with a selection on the significance of the transverse displacement with respect to the interaction point, $d_{x y} / \sigma\left(d_{x y}\right)>3$. The efficiency of the vertexing requirement, computed on a sample of $B^{ \pm} \rightarrow J / \psi K^{ \pm}$candidates, is shown in Figure 4 .

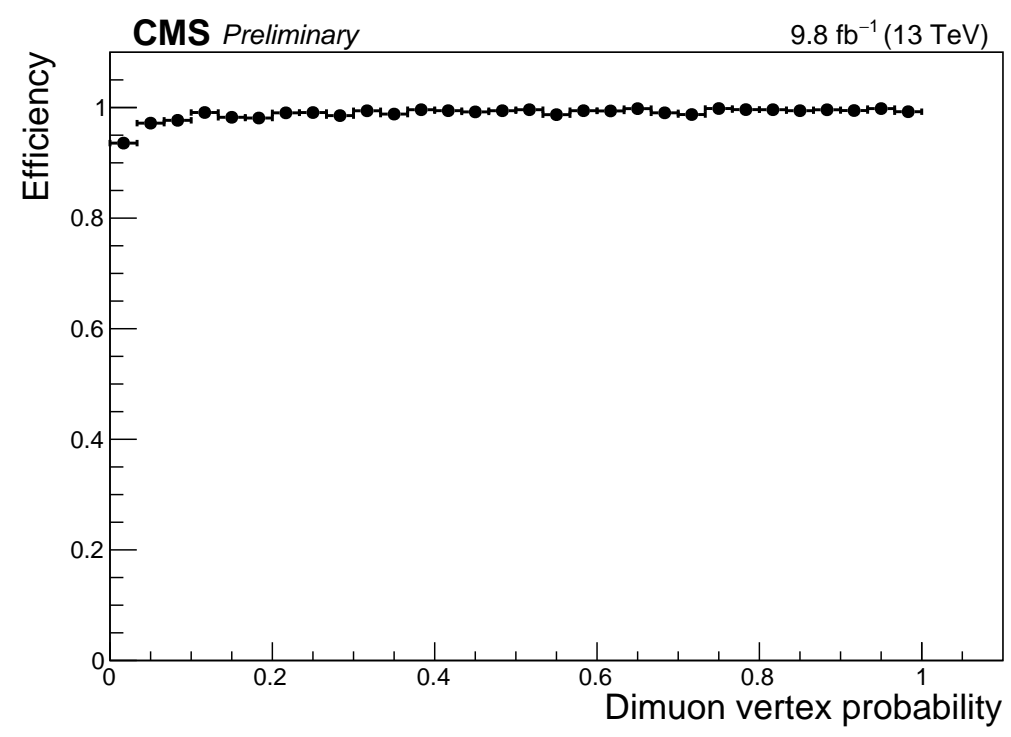

Figure 3: The efficiency of the High-Level Trigger requirement of a vertex between two muons, with no displaced topology, is shown against the offline dimuon vertex probability. Figure from [5].

\section{Conclusions}

The techniques introduced during LHC Run 2 in the CMS triggers used to collect events for B-physics analyses have been described. The efficiency of the improved trigger selections are 


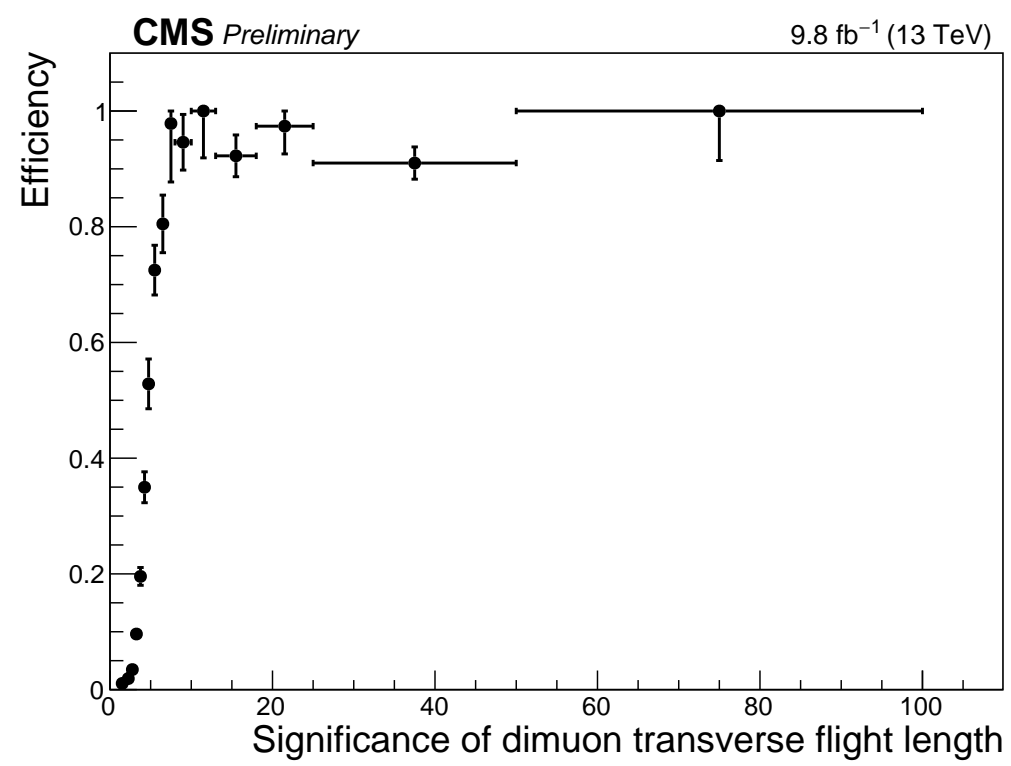

Figure 4: The efficiency of the High-Level Trigger requirement of a displaced vertex between two muons, used to select B-hadron decays, is shown against the significance of dimuon transverse flight length. In the online vertexing requirement, the threshold on the significance of the transverse displacement is set to $d_{x y} / \sigma\left(d_{x y}\right)>3$. Figure from [5].

computed on data collected during the 2017 run, and the results have been shown in this report. These results show that the newly introduced selections manage to reduce the rate of collected events, to fit in the available resources, with minimal impact on the efficiency on signal events.

\section{References}

[1] CMS Collaboration, The CMS trigger system, JINST 12 (2017), P01020.

[2] CMS Collaboration, The CMS experiment at the CERN LHC, JINST 3 (2008), S08004.

[3] CMS Collaboration, Level-1 muon trigger performance in 2017 data and, report: CMS-DP-2017-041, https://cds.cern.ch/record/2286327.

[4] CMS Collaboration, Dimuon Level-1 invariant mass in 2017 data, report: CMS-DP-2018-002, https://cds.cern.ch/record/2305543.

[5] CMS Collaboration, 2017 B-Physics trigger efficiencies, report: CMS-DP-2018-014, https://cds.cern.ch/record/2316914. 